\title{
Clinical implications and characterization of 1 casumat Group A Streptoccoccus infections in adults with cystic fibrosis
}

Kate Skolnik', Austin Nguyen², Ranjani Somayaji1,2, Christina S. Thornton², Barbara Waddell ${ }^{2}$, Michael G. Surette ${ }^{3,4,5}$, Harvey R. Rabin ${ }^{1,2}$ and Michael D. Parkins ${ }^{1,2^{*}}$

\begin{abstract}
Background: Persistent airway infection is a hallmark feature of cystic fibrosis (CF). However, increasingly it has been observed that non-classical pathogens may transiently infect CF lower airways. Streptococcus pyogenes (Group A Streptococcus; (GAS)) is an uncommon but potentially dangerous cause of community-acquired pneumonia. Our aim was to determine the incidence, natural history, and clinical impact of GAS infections in CF and phenotypically and genotypically characterize the isolates.
\end{abstract}

Methods: We retrospectively evaluated the Calgary Adult CF Clinic biobank to identify adults with at least one GAS isolate. Patient demographics, medical and pulmonary exacerbation (PEx) histories were evaluated. The primary outcome was PEx occurrence at incident GAS culture. Secondary outcomes evaluated were changes in lung function and PEx frequency following GAS isolation. Isolates were assessed for extra-cellular virulence factor production capacity and ability to produce quorum sensing (Al-2). Isolates were genotyped using pulse-field gel electrophoresis (PFGE).

Results: Fifteen individuals who cultured GAS twenty times were identified. At the time of GAS isolation, $47 \%$ (7/15) of subjects experienced a PEx and half of these (4/7) were severe. Individuals were more likely to have a PEx at the time of the index GAS isolate compared to the preceding visit ( $R R=6.0,95 \% \mathrm{Cl} 0.82-43.0, p=0.08)$, particularly if GAS was the numerically dominant sputum pathogen ( $R R=6.5,95 \% \mathrm{Cl} 1.00-43.0, p=0.009$ ). There were no changes in PEx frequency or rate of lung function decline following GAS. None of the patients developed chronic airways infection, bacteremia, necrotizing pneumonia or empyema. Susceptibility was universal to common anti-Streptococcal antibiotics and anti-Pseudomonal antibiotics commonly used in CF, with the exception of azithromycin. GAS isolates varied in their production of protease, DNase, and Al-2 but these did not correlate with PEx, and none produced elastase, chrondrotin sulfatase or $\mathrm{H}_{2} \mathrm{O}_{2}$. One patient had prolonged carriage with the same isolate and two patients had isolates with similar PFGE patterns.

Conclusions: GAS was an uncommon lower respiratory pathogen of adults with CF. Identification of GAS in sputum was frequently associated with PEx, particularly when numerically dominant. However, transient GAS infection did not result in chronic infection nor appreciably change long-term disease trajectory.

Keywords: Cystic fibrosis, Streptococcus pyogenes, Group A Streptococci, GAS, Pulmonary exacerbation, Eradication, Emerging pathogen

\footnotetext{
* Correspondence: mdparkin@ucalgary.ca

${ }^{1}$ Departments of Medicine, The University of Calgary, Calgary, AB, Canada

${ }^{2}$ Departments of Microbiology, Immunology and Infectious Diseases, The

University of Calgary, 3330 Hospital Drive, NW, Calgary, AB, Canada

Full list of author information is available at the end of the article
} 


\section{Background}

Cystic fibrosis (CF) is the most common lethal autosomal recessive disease amongst Caucasians [1]. Typically affected organs include the sinuses, lungs, gastrointestinal system and the male reproductive system. Pulmonary complications (both acute and chronic) are the primary cause of morbidity and mortality in the adult CF population [2, 3]. CF lungs are classically characterized by viscous secretions as well as impaired mucociliary clearance $[4,5]$. These factors compromise airway clearance creating an optimal environment for bacterial colonization, inflammation, chronic infection, and eventually, bronchiectasis.

The cultured microbiome of the CF respiratory system is well-characterized and unique from other forms of chronic lung disease. Nevertheless, our understanding of lung microbiology in the CF population continues to evolve. Traditional perceptions focused on chronic colonization with classical CF pathogens such as Pseudomonas aeruginosa, Staphylococcus aureus, Haemophilus influenzae, and Burkholderia cepacia complex [6]. Other microorganisms such as Stenotrophomonas maltophilia, Achromobacter species, and mycobacteria have been identified as emerging CF pathogens in the last two decades $[6,7]$. Whereas traditional pathogens like $P$. aeruginosa and B. cenocepacia have clearly been associated with adverse outcomes, the clinical implications are less clear with many emerging pathogens [8-10]. Furthermore, it is apparent that CF airways disease may exist in both stable (classical CF pathogens) and dynamic states (non-classical pathogens whose presence in the CF airways disease is usually only temporary). How these transient infections affect CF outcomes is entirely unknown. Consequently, clinicians are posed with unique management challenges when these atypical microbes are isolated from the lower respiratory tracts of CF patients.

One such organism is Group A Streptococcus (Streptococcus pyogenes), a common human pathogen that is rarely observed from CF lungs. S. pyogenes are $\beta$-hemolytic Gram positive cocci and facultative anaerobes. Its ability to cause hemolysis on blood agar plates allows it to be distinguished from other more indolent streptococci [11].

Furthermore, GAS has numerous virulence factors that enable it to evade host immune defenses, colonize epithelial surfaces, and cause infection [12, 13].

GAS can cause a variety of different infections with a spectrum of disease severity ranging from mild to invasive and life threatening [14]. While it most often manifests as pharyngeal, soft tissue or skin infections, it can rarely manifest as a respiratory infection. Although GAS only accounts for a small proportion of community acquired pneumonia in the general population, it tends to cause more severe and aggressive pulmonary infections and often manifest as empyema [14]. The prevalence, natural history and clinical effect of GAS in the CF population are unknown. Herein we set out to determine the natural history of GAS and outcomes associated with GAS infection in adults with CF. Furthermore, we sought to characterize GAS to determine if genotypic or phenotypic features were associated with disease.

\section{Methods}

Population

We retrospectively evaluated the Calgary Adult CF Clinic Biobank (CACFB; a prospectively collected and inventoried repository of every bacterial isolate from every CF sputum sample collected from each clinical encounter since 1978). Patients provide prospective consent for collection and research at enrollment into the clinic. Ethics for the collection and analysis was granted by the Conjoint Health Research Ethics Board (E-23087). Patients who cultured GAS at least once were included. There were no exclusion criteria other than age under 18 years.

\section{Clinical data collection}

The study design was retrospective and encompassed the two years preceding and two years following each GAS isolation. We collected baseline patient demographic information, dynamic spirometry data, exacerbation data, medications at the time of GAS isolate, and the presence of CF and non CF-related co-morbidities. Quantitative microbiology (reported as colony forming unit (CFU)/ml of sputum) was performed on each sample as is standard of care within our institution.

The primary outcome was the occurrence of pulmonary exacerbation (PEx) at the time of index GAS isolate compared to the preceding clinical visit. A PEx was defined based on a retrospective chart evaluation to identify documentation which met Fuchs' criteria and for which acute antibacterial therapy was prescribed [15]. A severe PEx was one requiring intravenous antibiotics and/or hospitalization. Secondary outcomes included: (1) change in predicted forced expiratory volume in one second $\left(\mathrm{FEV}_{1}\right)$ at the time of GAS identification versus the last clinic visit; (2) PEx frequency in individuals before and after the first GAS isolate; (3) rate of $\mathrm{FEV}_{1}$ decline before and after GAS, (4) severe complications of GAS infection; and (5) rate of progression to chronic infection.

\section{Characterization of GAS}

Genotyping and phenotyping were carried out on viable GAS isolates. GAS were genotyped using pulsed field gel electrophoresis (PFGE) to assess for clonality using the protocol of Sibley et al. [16]. Restriction digestion was performed independently with both SmaI and ApaI. PFGE profiles were compared using BioNumerics 
Version 7.0 (Applied Maths, Austin TX). Strains that had banding patterns $\geq 80 \%$ identical were a priori considered related, conforming to the Tenover criteria, where isolates with 1 to 3 band differences were still considered related [17]. Dendrograms were generated at $2.0 \%$ position tolerance using the unweighted pairgroup method with arithmetic mean (UPGMA) and the Sørensen-Dice similarity coefficient.

Antibiotic susceptibility testing was performed as per Thornton et al. [18]. Briefly, colonies from a 24-hour incubation on Columbia Blood Agar (CBA) were suspended in $0.85 \%$ saline solution to a 0.5 McFarland standard and spread on the Mueller-Hinton Blood Agar (MHBA) plates with a sterile cotton swab. Antibiotic discs were obtained from Oxoid (Nepean, Ontario) and stored at $4{ }^{\circ} \mathrm{C}$ until use. Antibiotics used in this investigation are those commonly used in $\mathrm{CF}$ and those frequently prescribed for GAS infection. The following antimicrobial discs were stamped on with a disc dispenser or manually placed: penicillin $\mathrm{G}(\mathrm{P}, 10 \mathrm{U})$, ceftriaxone (CRO, $30 \mu \mathrm{g})$, ceftazidime (CAZ, $30 \mu \mathrm{g})$, azithromycin (AZM, $15 \mu \mathrm{g}$ ), erythromycin (E, $15 \mu \mathrm{g}$ ), clindamycin (CDA, $2 \mu \mathrm{g}$ ), and levofloxacin (LEV, $15 \mu \mathrm{g}$ ). The "D-test" for inducible clindamycin resistance was performed. Plates were incubated for 20 to $24 \mathrm{~h}$ at $37^{\circ} \mathrm{C}$ with $5 \%$ carbon dioxide $\left(\mathrm{CO}_{2}\right)$ after which zone diameters were measured and compared to established breakpoints to determine susceptible (S), intermediate (I) and resistant (R) status as per CLSI guidelines $[19,20]$.

There were six exo-enzyme assays of GAS virulence factors capable of breaking down host products to aid in bacterial survival performed using cultures grown at $37{ }^{\circ} \mathrm{C}$ with $5 \% \mathrm{CO}_{2}$; each exo-enzyme assay involved the creation of a media with specific materials to test for the virulence factor as per the methods of Grinwis et al. [21]. Production of virulence factors was scored as positive (production of the factor) or negative (no evidence of this particular factor).

Autoinducer-2 (A1-2) is a universal quorum sensing molecule enabling interspecies signaling and communication. Cell free supernatants of each isolates were screened using a Vibrio harveyi bioassay for detection of the AI-2 using the methods of Grinwis et al. [21, 22]. In brief, overnight cultures of GAS were incubated in Todd-Hewitt-Yeast broth with $0.5 \%$ yeast extract (Bacto) for $24 \mathrm{~h}$ at $37{ }^{\circ} \mathrm{C}$ with $5 \% \mathrm{CO} 2.1 \mathrm{ml}$ was transferred to a $1.5 \mathrm{ml}$ tube and centrifuged at $2300 \mathrm{~g}$ for $5 \mathrm{~min}$ and filtered through $0.2 \mathrm{um}$ membrane. $400 \mathrm{ul}$ was stored at $-20{ }^{\circ} \mathrm{C}$ until use. The supernatant was mixed with the Vibrio harveyi MM32 reporter strain (luxS-, luxN-, deficient in production of AI-2 but sensitive to its production) and grown for $24 \mathrm{~h}$ in ToddHewitt- Yeast broth at $30{ }^{\circ} \mathrm{C}$. At 24 h luciferase production (counts per second) was measured using a Wallac
Victor2 microplate reader (Perkin Elmer, Waltham, MA) and reported as positive if $>2.5$ fold-above the negative control. All assays were performed in triplicate and for statistical analysis, Tukey's multiple comparison test was performed using Prism 5.0.

\section{Statistical analysis}

Demographic data was analyzed using descriptive statistics. Chi-Squared testing was used to determine the relative risk (RR) of PEx at the time of GAS isolation compared to the preceding visit as well as to determine if various clinical or microbiological factors affected the odds of PEx at GAS isolation. Wilcoxon rank- sum tests were used to compare the PEx rates and rate of FEV change in the 2 years before and 2 years following the index GAS isolates. Nonparametric tests were applied given the small sample size. Analysis was performed with STATA/IC 13.1 software (Stata- Corp, TX, USA).

\section{Results \\ Population characteristics}

Between 1978 and 2013, there were fifteen individuals from a cohort of 318 adults with CF $(4.7 \%)$ who had GAS isolated from their sputum. Thirteen patients cultured GAS only once, and two patients had repeated cultures for a total of 20 GAS isolates present within the CACFB. One patient (Patient 6) had three consecutive sputum samples growing GAS over 161 days, but subsequently cleared. Another patient (Patient 15) had intermittent GAS in their sputum on four separate occasions spanning 8.6 years. No patient became a chronic GAS carrier.

There were a comparable number of men and women ( 8 and 7, respectively) with a median age of 26 at the time of first GAS isolate (IQR 19 to 33 years) (Table 1). The majority had baseline airflow obstruction (86 \%), with a median $\mathrm{FEV}_{1}$ percent predicted of 50 \% (IQR 35 to 82.6) (Tables $1 \&$ 2). Most individuals were lifelonglong nonsmokers and had multi-organ CF disease in addition to bronchiectasis (Table 1). At the time of incident culture, half the group (7/15) was on chronic suppressive antibiotics (inhaled tobramycin or oral azithromycin) and a similar number received long-acting inhalers (inhaled corticosteroid, long acting beta agonist, or a combination ICS/LABA) (Table 1). Only one third were using an inhaled mucolytic and/or hypertonic saline (Table 1). Information regarding frequency, quality and nature of chest physiotherapy could not be reliably ascertained.

\section{Primary outcome - risk of pulmonary exacerbation}

At the time of first GAS isolation, $47 \%$ (7/15) of individuals experienced a PEx and approximately half of these (4/7) were severe. Of all encounters in which GAS was 
Table 1 Baseline demographics of adult cystic fibrosis patients with known S. pyogenes Sputum isolates

\begin{tabular}{|c|c|c|c|c|c|c|c|c|c|c|c|c|c|}
\hline Patient & $\mathrm{Age}^{\mathrm{a}}$ & Sex & Mutation 1 & Mutation 2 & $\begin{array}{l}\text { Pancreatic } \\
\text { Status }\end{array}$ & $\begin{array}{l}\text { Current } \\
\text { Smoker }\end{array}$ & $\begin{array}{l}\text { Baseline FEV }{ }^{\prime}(L)^{b} \\
\text { (\% predicted) }\end{array}$ & $\begin{array}{l}\text { Baseline FVC }(L)^{c} \\
\text { (\% predicted) }\end{array}$ & $\mathrm{TIS}^{\mathrm{d}}$ & AZM $^{\mathrm{e}}$ & $\operatorname{LABA}^{f}$ & $\mathrm{ICS}^{\mathrm{g}}$ & $\begin{array}{l}\mathrm{CF}^{\mathrm{h}} \mathrm{Co}- \\
\text { morbidities }\end{array}$ \\
\hline 1 & 19 & $M$ & F508del & F508del & I & $\mathrm{N}$ & $2.62(74)$ & $4.09(100)$ & Y & $N$ & $N$ & $N$ & DIOS, B \\
\hline 2 & 19 & M & F508del & $3849+1 G->A$ & I & $\mathrm{N}$ & $3.07(71)$ & - & $\mathrm{N}$ & $\mathrm{N}$ & $\mathrm{N}$ & $\mathrm{N}$ & Liver, S \\
\hline 3 & 34 & M & F508del & E56K & S & $\mathrm{N}$ & $3.87(106)$ & $4.98(115)$ & N & N & N & $N$ & None \\
\hline 4 & 18 & $F$ & M1101K & M1101K & । & $\mathrm{N}$ & $2.22(71)$ & $3.06(93)$ & Y & $\mathrm{N}$ & N & $N$ & \\
\hline 5 & 24 & $\mathrm{~F}$ & F508del & F508del & I & Unknown & $1.19(35)$ & $1.84(47)$ & N & $N$ & $N$ & $N$ & DIOS \\
\hline 6 & 28 & $\mathrm{~F}$ & F508del & F508del & 1 & $\mathrm{~N}$ & $2.39(89)$ & $3.72(122)$ & N & N & N & $N$ & IGT,DIOS \\
\hline 7 & 38 & $\mathrm{~F}$ & F508del & $\mathrm{R} 347 \mathrm{H}$ & S & Unknown & $2.15(77)$ & $3.14(95)$ & N & $N$ & $N$ & $N$ & S \\
\hline 8 & 48 & M & F508del & F508del & I & $\mathrm{N}$ & $2.30(54)$ & $4.79(90)$ & Y & Y & Y & $N$ & IGT \\
\hline 9 & 19 & M & $1717-1 \mathrm{G}->\mathrm{A}$ & Unknown & S & $\mathrm{N}$ & $3.73(82)$ & $4.55(85)$ & $N$ & $N$ & $N$ & $N$ & S \\
\hline 10 & 21 & M & F508del & F508del & I & Y & $2.63(40)$ & $3.61(84)$ & N & $N$ & $N$ & $N$ & DIOS \\
\hline 11 & 33 & F & F508del & R334W & I & $\mathrm{N}$ & $1.11(71)$ & $2.34(74)$ & $N$ & $N$ & $N$ & $N$ & DIOS \\
\hline 12 & 26 & M & G542x & Unknown & I & Y & $2.96(69)$ & $4.99(100)$ & $N$ & Y & Y & $N$ & CFRD \\
\hline 13 & 26 & $F$ & Unknown & Unknown & I & $N$ & $2.80(88)$ & $4.10(108)$ & Y & $N$ & $N$ & $N$ & $\begin{array}{l}\text { CFRD,B, S, } \\
\text { DIOS, }\end{array}$ \\
\hline 14 & 24 & M & F508del & F508del & I & $N$ & $3.84(84)$ & $5.80(115)$ & Y & $N$ & $N$ & Y & Liver \\
\hline 15 & 28 & $\mathrm{~F}$ & M1101K & M1101K & I & N & $2.67(91)$ & 3.37 (101) & $N$ & $N$ & $\mathrm{~N}$ & $\mathrm{~N}$ & $B, S$ \\
\hline
\end{tabular}

$I$ insufficient, $S$ sufficient, $Y$ yes, $N$ no

${ }^{\text {a }}$ Age at time of first $S$. pyogenes isolation.

${ }^{\mathrm{b}}$ Baseline FEV 1 forced expiratory volume in one second recorded 2 years prior to the index GAS isolate

c Baseline FVC forced volume capacity recorded 2 years prior to the index GAS isolate

d TIS Chronic inhaled tobramycin therapy

e $A Z M$ Chronic azithromycin

${ }^{f} \angle A B A$ Long-acting beta agonist

${ }^{9}$ ICS Inhaled corticosteroid

${ }^{\mathrm{h}}$ CF Comorbidities, DIOS Distal Ileal Obstruction Syndrome, B osteoporosis, Liver liver disease, S sinus disease, IGT Impaired Glucose Tolerance, CFRD CF related diabetes

identified, $45 \%(9 / 20)$ were associated with a PEx; $44 \%$ (4/9) of which were severe (Table 3). Relative to the preceding clinical visit, patients had a trend towards increased PEx risk at incident GAS isolation $(\mathrm{RR}=6.0$, $95 \%$ CI $0.82-43.0, p=0.08$ ). If GAS was the numerically dominant organism in the collected sputum, risk was further increased ( $\mathrm{RR}=6.5,95 \%$ CI 1.00-43.0, $p=$ 0.009); however, there was no specific log threshold for GAS above which PEx was more likely. Risk of PEx was not affected by the individual's age, sex, severe airflow obstruction at the preceding visit (as defined by \% predicted $\mathrm{FEV}_{1}<40$ ), or presence of specific chronic cultured microorganisms (as defined by Leeds Criteria) [23]. Furthermore, the risk of PEx was not influenced by the use of chronic antibiotics, inhalers, mucolytics or hypertonic saline (data not shown).

\section{Exacerbation frequency and lung function}

Of the 76 PEx during the study period, 38 occurred before and 29 after the first GAS isolate, respectively. The median number of PEx per patient was 2.5 (IQR 0 to 5) in the two years preceding index GAS isolate, compared to 2 (IQR 0 to 3 ) in the two following years. GAS did not increase the frequency of PEx in the two years after the index GAS isolate compared to the two preceding years $(\mathrm{RR}=1.07,95 \% \mathrm{CI} 0.71$ to $1.63, p=0.77)$; the frequency of severe PEx was also unchanged $(\mathrm{RR}=1.04$, $95 \%$ CI 0.5 to 2.13, $p=1.00)$. In fact, $60 \%(9 / 15)$ of individuals had a lower PEx frequency after the first GAS isolate.

Only $15 \%(2 / 13)$ of subjects had a significant decrease in their $\operatorname{FEV}_{1}(\geq 10 \%)$ at incident GAS isolate compared to the last visit and a minority of samples collected at any time with GAS $(2 / 17)$ were associated with a decreased $\mathrm{FEV}_{1}$. Furthermore, the rate of $\mathrm{FEV}_{1}$ decline did not significantly differ in the two years following the index isolate compared to the two preceding years; this was true for both the decline in absolute $\mathrm{FEV}_{1},(p=0.27)$ and $\%$ predicted $\operatorname{FEV}_{1}(p=0.72)$.

\section{Clinical outcomes}

Bacteremia, necrotizing pneumonia, and empyema were not observed with any of the GAS isolates. In the two years post index isolate, no individuals required initiation of chronic oxygen therapy or listing for lung transplant. 
Table 2 S.pyogenes sputum isolates and exacerbation status in adult cystic fibrosis patients

\begin{tabular}{|c|c|c|c|c|c|c|c|}
\hline Isolate & Patient $^{\mathrm{a}}$ & Date of Isolation & $\mathrm{CFU}^{\mathrm{b}}$ & GAS Most Abundant & Reduced FEV & $P E x^{d}$ & Chronic Bacterial Infections \\
\hline 1 & 1 & 08-1997 & $10^{6}$ & $\mathrm{~N}$ & $\mathrm{~N}$ & $\mathrm{~N}$ & PA \\
\hline 2 & 2 & 04-2010 & $10^{4}$ & N & Y & $Y^{a}$ & None \\
\hline 3 & 3 & 08-2013 & $10^{4}$ & Y & $\mathrm{N} / \mathrm{l}$ & Y & None \\
\hline 4 & 4 & 09-2010 & $10^{6}$ & Y & $\mathrm{N}$ & $\mathrm{N}$ & PA, MSSA \\
\hline 5 & 5 & 09-2010 & $10^{7}$ & Y & $\mathrm{N} / \mathrm{I}$ & Y & MSSA, HI \\
\hline 6 & 6 & $12-2001$ & $10^{7}$ & Y & $\mathrm{N}$ & $\mathrm{N}$ & MSSA \\
\hline 7 & 6 & 02-2002 & $10^{7}$ & Y & N & N & None \\
\hline 8 & 6 & 05-2002 & $10^{7}$ & Y & N & $Y^{a}$ & None \\
\hline 9 & 7 & 07-2006 & $10^{7}$ & Y & Y & Y & MSSA, HI \\
\hline 10 & 8 & 07-2012 & $10^{5}$ & $\mathrm{~N}$ & $\mathrm{~N}$ & $\mathrm{~N}$ & PA \\
\hline 11 & 9 & 10-1995 & $10^{8}$ & Y & N & $Y^{a}$ & MSSA, HI \\
\hline 12 & 10 & 05-1999 & $10^{7}$ & $N$ & $\mathrm{~N}$ & $\mathrm{~N}$ & MSSA, BC \\
\hline 13 & 11 & $01-2001$ & $10^{7}$ & Y & $\mathrm{N}$ & $N$ & MSSA \\
\hline 14 & 12 & $10-2012$ & $10^{5}$ & Y & $\mathrm{N}$ & N & PA \\
\hline 15 & 13 & $11-2012$ & $10^{6}$ & Y & N & Y & PA \\
\hline 16 & 14 & $11-2006$ & $10^{7}$ & Y & N & $\mathrm{N}$ & PA, MSSA \\
\hline 17 & 15 & $10-2000$ & $10^{6}$ & $\mathrm{~N}$ & $\mathrm{~N}$ & $N$ & PA, MSSA \\
\hline 18 & 15 & $11-2002$ & $10^{5}$ & $\mathrm{~N}$ & $\mathrm{~N}$ & Y & None \\
\hline 19 & 15 & 02-2009 & $10^{6}$ & N & N & Y & None \\
\hline 20 & 15 & 05-2009 & $10^{3}$ & N & N & N & None \\
\hline
\end{tabular}

${ }^{a}$ Refer to Table 1

${ }^{\mathrm{b}}$ CFU colony forming units/ml of sputum

C Reduced FEV1 reduction in reduction in $\mathrm{FEV}_{1}$ by $>10 \%$ at time of visit compared to baseline $\mathrm{FEV}_{1}$

d PEx pulmonary exacerbation as defined by Fuch's Criteria and the need for antibiotics

* Severe PEx pulmonary exacerbation requiring hospitalization and/or parenteral antibiotics

e PA Pseudomonas aeruginosa, MSSA Methicillin sensitive S. aureus, HI Haemophilus influenzae, Bc Burkholderia cenocepacia

$Y$ yes, $N$ no, $N / /$ no information, $N / I$ no information

\section{Phenotypic and genotypic characterization of GAS}

Antibiotic susceptibility testing data was available on all isolates to penicillin. Of the 20 isolates available in the biobank, 11 were viable for further phenotypic assessment and were compared against GAS ATCC 19615. All viable GAS strains were sensitive to levofloxacin, penicillin G, and ceftriaxone (Table 3). Azithromycin resistance was rare $(8 \%)$, and not observed in patients on chronic macrolide therapy. CLSI breakpoints for GAS do not exist, however ceftazidime (an agent commonly used in CF) demonstrated considerably inferior anti-GAS activity (median KB zone of $33 \mathrm{~mm}$ ). No clindamycin resistance, or inducible resistance with the D-test was observed. The majority (10/11) of GAS isolates produced DNAse and only one-quarter (3/11) produced elastase (Table 3). None of the GAS isolates produced protease, chondroitin sulfatase, or hydrogen peroxide (Table 3). AI-2 production was observed from all but one GAS isola te. There was no association between the production of particular virulence factors and the occurrence of PEx at isolation (data not shown).
Genotypic assessment of strain relatedness to assess for natural history of infection and potential for patientpatient spread was evaluated. PFGE profiles of the 11 viable isolates from the biobank and a control strain ATCC 19615 were performed. Patient A004 isolated the same strain of GAS over three months demonstrating persistent carriage of a single isolate was possible. Two patients A004 and A143 had isolates with the same PFGE profile with both SmaI (Fig. 1) and ApaI (not shown), although these isolates were collected three years apart suggesting patient-patient transmission was not a factor.

\section{Discussion}

Our study is the first to investigate the prevalence of GAS and its clinical effects in the adult CF population. $S$. pyogenes is a common organism that can affect healthy individuals of any age [14]. While GAS may transiently colonize the upper respiratory tract as a commensal organism (as is the case in 15 to $20 \%$ of healthy children), it has also proven itself as a major human 
Table 3 Antibiotic susceptibility and virulence factors of S. pyogenes sputum isolates from adult cystic fibrosis patients

\begin{tabular}{|c|c|c|c|c|c|c|c|c|c|c|c|c|c|}
\hline \multirow[t]{2}{*}{ Patient } & \multirow[t]{2}{*}{ Iso-late } & \multicolumn{6}{|c|}{ Antibiotic susceptibility } & \multicolumn{6}{|c|}{ Virulence Factor Production ${ }^{\mathrm{b}}$} \\
\hline & & PEN & LVX & $C A Z^{a}(\mathrm{~mm})$ & CRO & $\mathrm{CLI}$ & $\overline{A Z M}$ & Protease & Elastase & $\mathrm{H}_{2} \mathrm{O}_{2}$ & DNAse & Chondroitin Sulfatase & $\overline{\mathrm{Al}-2}$ \\
\hline 1 & 1 & S & S & 38 & S & S & S & $P$ & N & N & $P$ & N & N \\
\hline 2 & 2 & S & - & - & - & S & - & - & - & - & - & - & - \\
\hline 3 & 3 & S & - & - & - & - & - & - & - & - & - & - & - \\
\hline 4 & 4 & S & S & 37 & S & S & S & $\mathrm{N}$ & N & $\mathrm{N}$ & P & $N$ & P \\
\hline 5 & 5 & S & S & 33 & S & S & S & P & $N$ & $N$ & P & $N$ & P \\
\hline 6 & 6 & S & - & - & - & - & - & - & - & - & - & - & - \\
\hline 6 & 7 & S & - & - & - & - & - & - & - & - & - & - & - \\
\hline 6 & 8 & S & - & - & - & - & - & - & - & - & - & - & - \\
\hline 7 & 9 & S & S & 25 & S & $S$ & S & $\mathrm{N}$ & $N$ & $N$ & P & $N$ & P \\
\hline 8 & 10 & S & $S$ & 30 & S & S & $\mathrm{R}$ & $\mathrm{N}$ & $\mathrm{N}$ & $N$ & $P$ & $N$ & P \\
\hline 9 & 11 & S & S & 34 & S & $S$ & S & $N$ & $N$ & $N$ & $P$ & $N$ & P \\
\hline 10 & 12 & S & S & 34 & S & S & S & $\mathrm{N}$ & $N$ & $N$ & $P$ & $N$ & $P$ \\
\hline 11 & 13 & $S$ & S & 40 & $S$ & S & S & $\mathrm{N}$ & $N$ & $N$ & P & $N$ & $P$ \\
\hline 12 & 14 & S & - & - & - & $S$ & - & - & - & - & - & - & - \\
\hline 13 & 15 & S & S & 33 & S & $S$ & S & $\mathrm{N}$ & $N$ & $N$ & $\mathrm{~N}$ & $N$ & P \\
\hline 14 & 16 & S & - & - & - & - & - & - & - & - & - & - & - \\
\hline 15 & 17 & S & - & - & - & - & - & - & - & - & - & - & - \\
\hline 15 & 18 & S & - & - & - & - & - & - & - & - & - & - & - \\
\hline 15 & 19 & S & S & 35 & S & S & S & $\mathrm{N}$ & $N$ & $N$ & $P$ & $N$ & P \\
\hline 15 & 20 & S & S & 38 & S & $S$ & S & $\mathrm{N}$ & $N$ & $N$ & $P$ & $N$ & P \\
\hline ATCC & 19615 & $S$ & $S$ & 31 & $S$ & $S$ & S & $P$ & $\mathrm{~N}$ & $\mathrm{~N}$ & $P$ & $\mathrm{~N}$ & $P$ \\
\hline
\end{tabular}

PEN Penicillin susceptibility

LVX Levofloxacin susceptibility

CAZ Ceftazidime susceptibility

CRO Ceftriaxone susceptibility

CLI Clindamycin susceptibility

AZM Azithromycin susceptibility

(-) Indicates frozen isolate could not be successfully recovered.

$S$ Sensitive, / Intermediate, $R=$ Resistant

${ }^{a}=$ CLSI breakpoints do not exist for ceftazidime. Results reported as Kirby-Bauer zone sizes.

${ }^{\mathrm{b}}$ Production of virulence factors reported as strains producing those factors; $P$ positive (Strains produced this virulence factor), or $N=$ negative

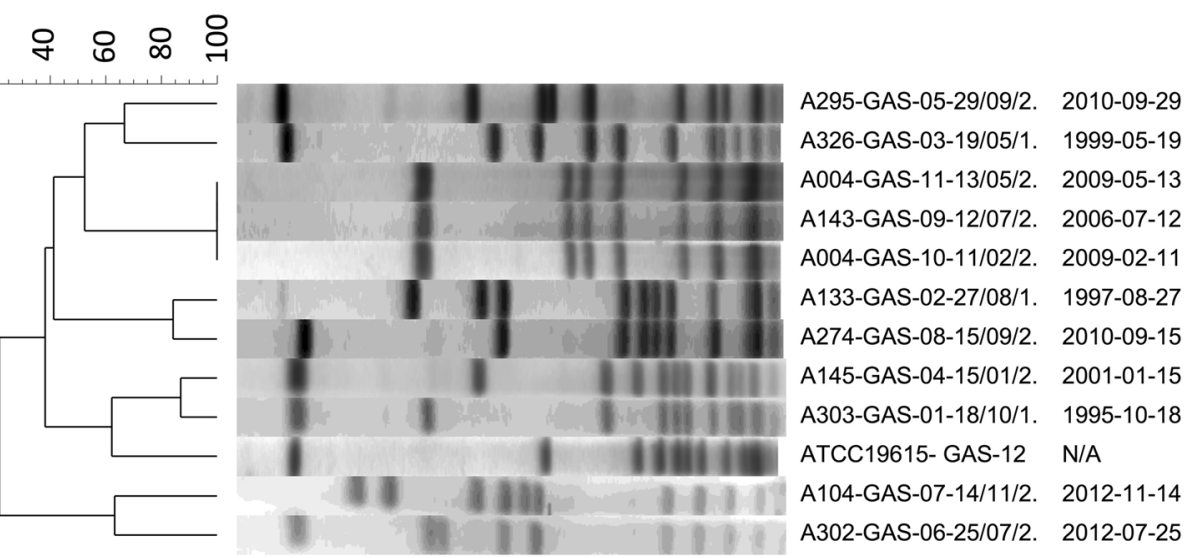

Fig. 1 Smal restriction digest pulse field gel electrophoresis of S. pyogenes recovered from CF sputum. $A=$ patient number, date indicated as year/month/day 
pathogen [24]. It is responsible for a broad spectrum of disease including pharyngitis, scarlet fever, rheumatic fever, cellulitis, necrotizing fasciitis, toxic shock syndrome, and pneumonia [14]. GAS infections can range from mild to life threatening; severe disease is not limited to those with chronic illness or immune compromise [14]. Globally, GAS is responsible for a significant burden of disease; every year it accounts for 110 million skin and soft tissue infections [11], 660000 cases of invasive infection [11] and over 294. 000 deaths [25].

From a pulmonary standpoint, GAS is an infrequent but important cause of pneumonia [26]. While it only accounts for a small percentage of community acquired pneumonia (CAP) in adults, S. pyogenes tends to cause more severe and invasive infection compared to common CAP pathogens [14]. Furthermore, GAS has a higher likelihood of resulting in necrotizing pneumonia, progression to empyema and/or hemorrhagic pleural effusion [13, 27]. Mortality rates for GAS pneumonia range between 20 and $38 \%$, which is similar to that of necrotizing fasciitis [13, 14].

Although S. pyogenes is a major global pathogen, little is known about its role in CF lung disease. Given the potential for GAS to cause exceptional virulence and the compromised innate immunity of CF lungs, we hypothesized that GAS may lead to adverse clinical outcomes in this population. There is a paucity of GAS epidemiologic data in the CF literature, with prior studies reporting prevalence of $0.8 \%(2 / 258)$ [28] and $0.9 \%(4 / 495)$ [29], respectively. Over 34 years, we identified that five percent of patients isolated GAS on at least one occasion. However, the presence of GAS increased the risk of PEx relative to the preceding clinic visit, particularly if present as the numerically dominant sputum pathogen. This finding may warrant treating individuals with GAS in their sputum with anti-GAS treatments in order to potentially avoid an ensuing PEx. However, other factors including exacerbation of chronically infecting pathogens, and inter-current upper respiratory viral illnesses could also have contributed (although these factors were just as likely in comparator clinical visits).

Within CF, it is clear that mere culture status may not convey the entire story. Indeed, differential pathogenic potential has been observed with the expression of a number of phenotypic traits of classical CF pathogens including $P$. aeruginosa, $B c c$, and $S$. aureus. For example, compared to patients with chronic methicillin-sensitive $S$. aureus (MSSA) infection, those with chronic methicillin-resistant $S$. aureus (MRSA) have an increased risk of death [30]. Patients with MRSA have increased rates of lung function decline [31] and are less likely to recover lung function following PEx [32]. In patients with chronic $P$. aeruginosa infections, its conversion to a hyper-alginate producing, mucoid phenotype is associated with progressive decline in lung function, increased risk of hospitalization and reduced survival $[8,33-36]$. The opposite appears true in $B c c$ chronically infected patients, where mucoidy appears protective and patients with nonmucoid isolates experience an exaggerated rate of clinical decline [37, 38]. Even the ability to persist within the CF lung seems to be influenced by specific phenotypic traits of $P$. aeruginosa causing initial infections [39]. The phenotypes that are associated with these strains may themselves not be directly involved in disproportionate lung disease, but rather they may be an indirect marker. Accordingly, we sought to characterize easily assayable and important virulence traits within infecting GAS strains to determine if these factors disproportionally modified PEx risk.

The GAS isolated from CF airways were typical of GAS reported in other diseases [40]. We identified variable expression of virulence factors, which has previously been reported [41]. Of the limited virulence factors assessed, expression did not increase risk for occurrence of PEx at the time of isolation. Almost all GAS isolates produced $\mathrm{AI}-2$, a diffusible cell-cell signaling molecule enabling inter-species bacterial communication on cell density $[42,43]$. Either directly (GAS mediated primary effects) or indirectly (through induction of quorum sensing in patients chronically colonized with pathogens such as $P$. aeruginosa), GAS may be able to trigger a PEx [44].

The antibiotic sensitivity profile of our strains was similar to that of other S. pyogenes epidemiologic studies $[40,45]$, with the exception of a complete absence of clindamycin resistance in our few isolates. Interestingly, our study along with several small studies $[40,45]$, have not found high rates of macrolide or fluoroquinolone resistance, as reported in larger studies (despite their frequent use in $\mathrm{CF}$ ). Indeed, this may suggest that those GAS in CF are not unrecognized chronic endogenous lower respiratory tract flora in these individuals but rather newly acquired transient organisms not previously exposed to antibiotics. Importantly, our GAS strains were sensitive to antibiotics commonly used in the empiric treatment of CF PEx, although ceftazidime, an antibiotic commonly used in the empiric PEx management, should not be used where GAS is involved. Nearly all strains were sensitive to azithromycin; this raises the possibility that chronic azithromycin may suppress GAS and prevent initial colonization. Indeed, registry data suggests that $60-70 \%$ of $C F$ patients with chronic $P$. aeruginosa infection and $22 \%$ of CF patients without chronic $P$. aeruginosa receive chronic macrolide therapy and this may account for its low observed incidence in our cohort $[46,47]$.

PFGE has been shown to be similarly effective at differentiating commonly infecting clones of GAS as other established typing modalities including emm gene typing 
[48-50]. Using PFGE we demonstrated strain persistence in those patients with repeated positive cultures, rather than repeated new infections with different strains. We did identify two patients with the same GAS isolate by PFGE, but propose this was unlikely to be patient-patient spread. Whereas typical CF pathogens are rare and opportunistic of the general population, GAS commonly colonizes the upper respiratory tract in the general population and common strains persist in locals for extended period of times [51, 52]. Furthermore, GAS from these patients were identified $>2.5$ years apart with multiple negative cultures in the ensuing time period making CF patientpatient transmission biologically implausible.

Members of the genus Streptococcus have not traditionally been considered CF respiratory pathogens. However, using a combination of semi-selective agars and high density sampling, high rates of Viridans Group Streptococci (VGS) have universally been identified [53-55]. The Streptococcus anginosus group, in particular, is increasingly thought to have a role in CF and its emergence as numerically dominant organism has been observed in a large subset of PEx [54]. Traditional clinical microbiology protocols have been developed to overlook VGS. However, GAS is easily identifiable as beta-hemolysis is a defining feature (present in $99 \%$ of isolates) [56], and as such is more likely to be distinguished from oropharyngeal streptococci using traditional culture techniques.

The role of bacteria in CF airways disease has been viewed through the lens of contributing to chronic progressive lung disease $[57,58]$. Indeed, when assessing traditional CF pathogens such as $P$. aeruginosa and $B$. cepacia complex, as well as emerging organisms such as S. maltophilia, this model holds true. However, increasingly organisms not typically associated with CF airways disease including the Enterobactericeae, Pneumococcus, and GAS are seen to transiently colonize the airways $[8,59,60]$. The impact of these organisms on short-term and long-term outcomes are for the most part unknown. Given that transient colonization/infection with respiratory viruses has been shown to produce short term deleterious effects either through direct pathogenesis or indirectly through resident microflora, so too might transient bacterial pathogens [61]. Indeed, while an acute impact on patient well-being was observed with incident GAS infection, long term effects were not noted, nor should they be expected. This highlights the concept that emerging CF pathogens may not necessarily manifest as chronic infections, as is often seen with $P$. aeruginosa, $S$. aureus, and $B c c$.

The main limitations of the study are the retrospective design and wide confidence intervals relating to small sample size and few events. Despite trying to account for factors that could influence the risk of PEx, we were unable to control for differences in chest physiotherapy quality and frequency as well as adherence to medications. We also lacked documentation regarding potential confounding viral and/or environmental triggers for PEx. Future studies assessing potential short-term impact of transient airway colonizers are warranted based on the data herein.

\section{Conclusion}

GAS was an uncommon, transient lower respiratory pathogen in our population of adults with CF. In our cohort, identification of GAS in sputum, particularly as the dominant organism, was associated with an increased PEx risk. However, GAS colonization of the lower airways was transient and, as such, did not impact lung function and did not appreciably change the disease trajectory.

\section{Abbreviations}

AB: Agrobacterium minimal medium; Al-2: Auto inducer 2;

AZM: Azithromycin; BHI: Brain-heart infusion; CF: Cystic fibrosis; $\mathrm{CO}_{2}$ : Carbon dioxide; CFU: Colony forming units; CAP: Community acquired pneumonia; CAZ: Ceftazidime; CBA: Columbia blood agar; CDA: Clindamycin; CLSI: Clinial and Laboratory Standards Institute; CRO: Ceftriaxone; E: Erythromycin; $\mathrm{FEV}_{1}$ : Change in \% predicted forced expiratory volume in one second: GAS: Group A Streptococcus; PEx: Pulmonary exacerbation; ICS: Inhaled corticosteroid; I: Intermediate; KB: Kirby Bauer; LABA: Long acting beta agonist; LEV: Levofloxacin; MHBA: Mueller-Hinton blood agar; MSSA: Methicillin sensitive Staphylococcus aureus; OD: Optical denstity; P: Penicillin G; PFGE: Pulse field gel electrophoresis; R: Resistant; S: Susceptible; TSY: Trypticase soy yeast agar; UPGMA: Unweighted pair-group method with arithmetic mean.

\section{Competing interests}

MDP and MGS are supported through research grants from Cystic Fibrosis Canada and Gilead. MDP, HRR have performed advisory board duties for Gilead, Novartis, Roche and Vertex. None of these relate to the work contained herein and the authors declare that they have no competing interests.

\section{Authors' contributions}

KS was responsible for collection and analysis of all patient related data, and drafting the manuscript. AN, BW, CST performed GAS genotyping and phenotyping and assisted in the revision of the manuscript. RS assisted in the statistical analysis, data collection and revision of the manuscript. MGS, HRR and MDP were responsible for the conception of the project, supervision of the collection and analysis of patient and microbiologic data, and assisted with revising the manuscript. All authors read and approved the final manuscript.

\section{Acknowledgements}

We gratefully acknowledge the clinical staff of Calgary Laboratory Services for their participation in the collection and organization of the Calgary Adult CF Biobank.

\section{Author details \\ ${ }^{1}$ Departments of Medicine, The University of Calgary, Calgary, AB, Canada. ${ }^{2}$ Departments of Microbiology, Immunology and Infectious Diseases, The University of Calgary, 3330 Hospital Drive, NW, Calgary, AB, Canada. \\ ${ }^{3}$ Department of Medicine, McMaster University, Hamilton, ON, Canada. \\ ${ }^{4}$ Department of Biochemistry, McMaster University, Hamilton, ON, Canada. ${ }^{5}$ The Farncombe Family Digestive Health Research Institute, McMaster University, Hamilton, ON, Canada.}

Received: 12 August 2015 Accepted: 8 December 2015

Published online: 12 December 2015 


\section{References}

1. Hamosh A, FitzSimmons SC, Macek Jr M, Knowles MR, Rosenstein BJ, Cutting GR. Comparison of the clinical manifestations of cystic fibrosis in black and white patients. J Pediatr. 1998;132(2):255-9.

2. Ratjen F, Doring G. Cystic fibrosis. Lancet. 2003;361(9358):681-9.

3. Alicandro G, Frova L, Di Fraia G, Colombo C. Cystic fibrosis mortality trend in Italy from 1970 to 2011. J Cyst Fibros. 2015;14(2):267-74.

4. Guggino WB. Cystic fibrosis and the salt controversy. Cell. 1999;96(5):607-10

5. Houtmeyers E, Gosselink R, Gayan-Ramirez G, Decramer M. Regulation of mucociliary clearance in health and disease. Eur Respir J. 1999;13(5):1177-88.

6. Mahenthiralingam E. Emerging cystic fibrosis pathogens and the microbiome. Paediatr Respir Rev. 2014;15 Suppl 1:13-5.

7. Canadian Cystic Fibrosis Foundation. The Canadian cystic fibrosis registry: 2013 annual data report. 2015. p. 32-3.

8. Kerem E, Corey M, Gold R, Levison H. Pulmonary function and clinical course in patients with cystic fibrosis after pulmonary colonization with Pseudomonas aeruginosa. J Pediatr. 1990;116(5):714-9.

9. Fauroux B, Hart N, Belfar S, Boule M, Tillous-Borde I, Bonnet D, et al. Burkholderia cepacia is associated with pulmonary hypertension and increased mortality among cystic fibrosis patients. J Clin Microbiol. 2004:42(12):5537-41.

10. Kalish LA, Waltz DA, Dovey M, Potter-Bynoe G, McAdam AJ, Lipuma JJ, et al. Impact of Burkholderia dolosa on lung function and survival in cystic fibrosis. Am J Respir Crit Care Med. 2006;173(4):421-5.

11. Tan LK, Eccersley LR, Sriskandan S. Current view of haemolytic streptococcal infections. Curr Opin Infect Dis. 2014;27:155-64.

12. Cole JN, Barnett TC, Nizet V, Walker MJ. Molecular insight into invasive group A streptococcal disease. Nat Rev Microbiol. 2011;9(10):724-36.

13. Bisno AL, Brito MO, Collins CM. Molecular basis of group A streptococcal virulence. Lancet Infect Dis. 2003;3(4):191-200.

14. Muller MP, Low DE, Green KA, Simor AE, Loeb M, Gregson D, et al. Clinical and epidemiologic features of group a streptococcal pneumonia in Ontario, Canada. Arch Intern Med. 2003;163(4):467-72.

15. Fuchs HJ, Borowitz DS, Christiansen DH, Morris EM, Nash ML, Ramsey BW, et al. Effect of aerosolized recombinant human DNase on exacerbations of respiratory symptoms and on pulmonary function in patients with cystic fibrosis. The pulmozyme study group. N Engl J Med. 1994;331(10):637-42.

16. Sibley CD, Sibley KA, Leong TA, Grinwis ME, Parkins MD, Rabin HR, et al. The Streptococcus milleri population of a cystic fibrosis clinic reveals patient specificity and intraspecies diversity. J Clin Microbiol. 2010;48(7):2592-4.

17. Tenover FC, Arbeit RD, Goering RV, Mickelsen PA, Murray BE, Persing DH, et al. Interpreting chromosomal DNA restriction patterns produced by pulsed-field gel electrophoresis: criteria for bacterial strain typing. J Clin Microbiol. 1995;33(9):2233-9.

18. Thornton CS, Brown EL, Alcantara J, Rabin HR, Parkins MD. Prevalence and impact of Streptococcus pneumoniae in adult cystic fibrosis patients: a retrospective chart review and capsular serotyping study. BMC Pulm Med. 2015;15:49.

19. CLSI. Performance standards for antimicrobial disk susceptibility tests; approved standard-tenth edition, CLSI document M02-A10. 2009. Clinical and Laboratory Standards Institute.

20. CLSI. Performance standards for antimicrobial susceptibility testing; nineteenth informational supplement. In: (CLSI) CaLSI, editor. Clinical and laboratory standards institute. 2009. p. 1-156.

21. Grinwis ME, Sibley CD, Parkins MD, Eshaghurshan CS, Rabin HR, Surette MG. Characterization of Streptococcus milleri group isolates from expectorated sputum of adult patients with cystic fibrosis. J Clin Microbiol. 2010;48(2):395-401.

22. Surette MG, Miller MB, Bassler BL. Quorum sensing in Escherichia coli, Salmonella typhimurium, and Vibrio harveyi: a new family of genes responsible for autoinducer production. Proc Natl Acad Sci U S A. 1999:96(4):1639-44

23. Lee TWR, Brownlee KG, Conway SP, Denton M, Littlewood JM. Evaluation of a new definition for chronic Pseudomonas aeruginosa infection in cystic fibrosis patients. J Cystic Fibros. 2003;2:29-34.

24. Vincent MT, Celestin N, Hussain AN. Pharyngitis. Am Fam Physician. 2004;69(6):1465-70.

25. Carapetis JR, Steer AC, Mulholland EK, Weber M. The global burden of group A streptococcal diseases. Lancet Infect Dis. 2005;5(11):685-94.

26. Marston BJ, Plouffe JF, File Jr TM, Hackman BA, Salstrom SJ, Lipman HB, et al. Incidence of community-acquired pneumonia requiring hospitalization. Results of a population-based active surveillance study in Ohio The Community-Based Pneumonia Incidence Study Group. Arch Intern Med. 1997;157:1709-18.

27. Santagati M, Spanu T, Scillato M, Santangelo R, Cavallaro F, Arena V, et al. Rapidly fatal hemorrhagic pneumonia and group A Streptococcus serotype M1. Emerg Infect Dis. 2014;20(1):98-101.

28. Doern GV, Brogden-Torres B. Optimum use of selective plated media in primary processing of respiratory tract specimens from patients with cystic fibrosis. J Clin Microbiol. 1992;30(10):2740-2.

29. Burns JL, Emerson J, Stapp JR, Yim DL, Krzewinski J, Louden L, et al. Microbiology of sputum from patients at cystic fibrosis centers in the United States. Clin Infect Dis. 1998;27(1):158-63.

30. Dasenbrook EC, Checkley W, Merlo CA, Konstan MW, Lechtzin N, Boyle MP. Association between respiratory tract methicillin-resistant Staphylococcus aureus and survival in cystic fibrosis. JAMA. 2010;303(23):2386-92.

31. Dasenbrook EC, Merlo CA, Diener-West M, Lechtzin N, Boyle MP. Persistent methicillin-resistant Staphylococcus aureus and rate of FEV1 decline in cystic fibrosis. Am J Respir Crit Care Med. 2008;178(8):814-21.

32. Sanders DB, Bittner RC, Rosenfeld M, Hoffman LR, Redding GJ, Goss CH. Failure to recover to baseline pulmonary function after cystic fibrosis pulmonary exacerbation. Am J Respir Crit Care Med. 2010;182(5):627-32

33. Henry RL, Mellis CM, Petrovic L. Mucoid Pseudomonas aeruginosa is a marker of poor survival in cystic fibrosis. Pediatr Pulmonol. 1992;12(3):158-61.

34. Li Z, Kosorok MR, Farrell PM, Laxova A, West SE, Green CG, et al. Longitudinal development of mucoid Pseudomonas aeruginosa infection and lung disease progression in children with cystic fibrosis. JAMA. 2005;293(5):581-8.

35. Kosorok MR, Zeng L, West SE, Rock MJ, Splaingard ML, Laxova A, et al. Acceleration of lung disease in children with cystic fibrosis after Pseudomonas aeruginosa acquisition. Pediatr Pulmonol. 2001;32(4):277-87.

36. Emerson J, Rosenfeld M, McNamara S, Ramsey B, Gibson RL. Pseudomonas aeruginosa and other predictors of mortality and morbidity in young children with cystic fibrosis. Pediatr Pulmonol. 2002:34(2):91-100.

37. Silva IN, Ferreira AS, Becker JD, Zlosnik JE, Speert DP, He J, et al. Mucoid morphotype variation of Burkholderia multivorans during chronic cystic fibrosis lung infection is correlated with changes in metabolism, motility, biofilm formation and virulence. Microbiology. 2011;157(11):3124-37.

38. Zlosnik JE, Speert DP. The role of mucoidy in virulence of bacteria from the Burkholderia cepacia complex: a systematic proteomic and transcriptomic analysis. J Infect Dis. 2010;202(5):770-81.

39. Manos J, Hu H, Rose BR, Wainwright CE, Zablotska IB, Cheney J, et al. Virulence factor expression patterns in Pseudomonas aeruginosa strains from infants with cystic fibrosis. Eur J Clin Microbiol Infect Dis. 2013:32(12):1583-92.

40. Karaky NM, Araj GF, Tokajian ST. Molecular characterization of Streptococcus pyogenes group A isolates from a tertiary hospital in Lebanon. J Med Microbiol. 2014;63(Pt 9):1197-204.

41. Borek AL, Obszanska K, Hryniewicz W, Sitkiewicz I. Detection of Streptococcus pyogenes virulence factors by multiplex PCR. Virulence. 2012;3(6):529-33.

42. Marouni MJ, Sela S. The luxS gene of Streptococcus pyogenes regulates expression of genes that affect internalization by epithelial cells. Infect Immun. 2003;71(10):5633-9.

43. Siller M, Janapatla RP, Pirzada ZA, Hassler C, Zinkl D, Charpentier E. Functional analysis of the group A streptococcal luxS/Al-2 system in metabolism, adaptation to stress and interaction with host cells. BMC Microbiol. 2008:8:188.

44. Duan K, Dammel C, Stein J, Rabin H, Surette MG. Modulation of Pseudomonas aeruginosa gene expression by host microflora through interspecies communication. Mol Microbiol. 2003;50(5):1477-91.

45. Camara M, Dieng A, Boye CS. Antibiotic susceptibility of streptococcus pyogenes isolated from respiratory tract infections in dakar, senegal. Microbiol Insights. 2013;6:71-5.

46. http://www.cysticfibrosisdata.org/ReportsUS.html. Accessed May 152015.

47. http://www.cysticfibrosis.org.uk/media/598466/annual-data-report-2013jul14.pdf. Accessed April 202015.

48. Stepak P, Roberts MC, Goldoft M, Kobayashi J. Use of pulsed-field Gel electrophoresis for investigation of a cluster of invasive group a streptococcal illness - Spokane, Washington. MMWR Morb Mortal Wkly Rep. 1999:48(31):681-3. http://www.cdc.gov/mmwr/preview/mmwrhtml/ mm4831a1.htm. Accessed October 62015.

49 Silva-Costa C, Friães A, Ramirez M, Melo-Cristino J, The Portuguese Group for the Study of Streptococcal Infections. Differences between macrolide- 
resistant and - susceptible streptococcus pyogenes: importance of clonal properties in addition to antibiotic consumption. Antimicrob Agents Chemother. 2012;56(11):5661-6.

50. Carriço JA, Silva-Costa C, Melo-Cristino J, Pinto FR, de Lencastre H, Almeida JS, et al. Illustration of a common framework for relating multiple typing methods by application to macrolide- resistant streptococcus pyogenes. J Clin Microbiol. 2006;44(7):2524-32.

51. Steer AC, Law I, Matatolu L, Beall BW, Carapetis JR. Global emm type distribution of group A streptococci: systematic review and implications for vaccine development. Lancet Infect Dis. 2009;9:611-6.

52. Teatero S, McGeer A, Low DE, Li A, Demczuk W, Martin I, et al. Characterization of invasive group $B$ streptococcus strains from the greater Toronto Area, Canada. J Clin Microbiol. 2014;52(5):1441-7.

53. Maeda Y, Elborn JS, Parkins MD, Reihill J, Goldsmith CE, Coulter WA, et al. Population structure and characterization of viridans group streptococci (VGS) including Streptococcus pneumoniae isolated from adult patients with cystic fibrosis (CF). J Cyst Fibros. 2011;10(2):133-9.

54. Sibley CD, Grinwis ME, Field TR, Parkins MD, Norgaard JC, Gregson DB, et al. McKay agar enables routine quantification of the 'Streptococcus milleri' group in cystic fibrosis patients. J Med Microbiol. 2010;59(Pt 5):534-40.

55. Sibley CD, Parkins MD, Rabin HR, Duan K, Norgaard JC, Surette MG. A polymicrobial perspective of pulmonary infections exposes an enigmatic pathogen in cystic fibrosis patients. Proc Natl Acad Sci U S A. 2008;105(39):15070-5.

56. Yoshino M, Murayama SY, Sunaoshi K, Wajima T, Takahashi M, Masaki J, et al. Nonhemolytic Streptococcus pyogenes isolates that lack large regions of the sag operon mediating streptolysin S production. J Clin Microbiol. 2010;48(2):635-8.

57. Lipuma JJ. The changing microbial epidemiology in cystic fibrosis. Clin Microbiol Rev. 2010;23(2):299-323.

58. Surette MG. The cystic fibrosis lung microbiome. Ann Am Thorac Soc. 2014;11 Suppl 1:S61-5.

59. Barillova P, Tchesnokova V, Dubbers A, Kuster P, Peters G, Dobrindt U, et al. Prevalence and persistence of Escherichia coli in the airways of cystic fibrosis patients an unrecognized CF pathogen? Int J Med Microbiol. 2014;304(3-4):415-21.

60. Parkins MD, Floto RA. Emerging bacterial pathogens and changing concepts of bacterial pathogenesis in cystic fibrosis. J Cyst Fibros. 2015;14(3):293-304.

61. Etherington C, Naseer R, Conway SP, Whitaker P, Denton M, Peckham DG. The role of respiratory viruses in adult patients with cystic fibrosis receiving intravenous antibiotics for a pulmonary exacerbation. J Cyst Fibros. 2014;13(1):49-55

\section{Submit your next manuscript to BioMed Central and we will help you at every step:}

- We accept pre-submission inquiries

- Our selector tool helps you to find the most relevant journal

- We provide round the clock customer support

- Convenient online submission

- Thorough peer review

- Inclusion in PubMed and all major indexing services

- Maximum visibility for your research

Submit your manuscript at www.biomedcentral.com/submit 